Gynäkologische Endokrinologie 2014 · 12:137

DOI 10.1007/s10304-013-0598-7

Online publiziert: 4. Juli 2014

c) Springer-Verlag Berlin Heidelberg 2014

T. Strowitzki ${ }^{1}$ W. Küpker ${ }^{2}$ A A.O. Mueck ${ }^{3}$

${ }^{1}$ Gynäkologische Endokrinologie und Fertilitätsstörungen, Universitäts-Frauenklinik, Heidelberg

${ }^{2}$ Endometriosezentrum, Stadtklinik Baden-Baden

${ }^{3}$ Universitätsfrauenklinik Tübingen

\title{
Hormone und Krebs
}

die Women's Health Initiative vor mehr als 10 Jahren, die Untersuchungen zu den Einflüssen von Hormontherapien auf Krebserkrankungen ins Rollen gebracht hat. Eine Bewertung dieses umfassenden Themas durch namhafte Autoren hat sich deshalb diese Ausgabe von Gynäkologische Endokrinologie zur Aufgabe gemacht. Dabei beschränken wir uns nicht auf die Risikobewertung der Hormonersatztherapie (HRT), sondern greifen viele verschiedene Themen um Hormone und Krebs auf.

Wie wirkt sich die Pilleneinnahme auf das Krebsrisiko aus? Gibt es Unterschiede je nach Tumorerkrankungen? In welchem Verhältnis stehen protektive Einflüsse und schädliche Auswirkungen der Pille? Diesen Fragen gehen Schmidmayr et al. aus München nach.

Bei an Krebs erkrankten Frauen hat wegen der bedeutenden Fortschritte der onkologischen Therapie der Erhalt der Fertilität einen ganz neuen Stellenwert erlangt. $\mathrm{Zu}$ den Therapieoptionen der Fertilitätsprotektion zählen natürlich auch verschiedene hormonelle Behandlungen, die von der Gabe von Gonadotropin-Releasing-Hormon-Analoga bis zur direkten hormonellen Stimulation vor Methoden der assistierten Reproduktion reichen. Welche Therapieoptionen sind aber im Kontext welcher Tumorentitäten ver- tretbar? Hier herrscht große Unsicherheit. Lawrenz $u$. Nawroth gehen daher auf diese Behandlungsformen ein.

Mueck u. Seeger nehmen sich die Risikobewertung von Brustkrebs und HRT vor. Dieses Thema ist sicher das meist diskutierte der letzten 10 Jahre. Klinische und experimentell-biologische Daten helfen bei einer realistischen Bewertung der Studienlage.

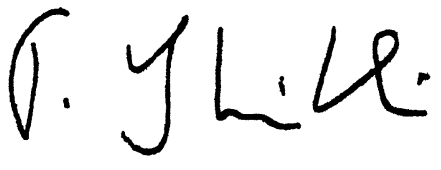

T. Strowitzki

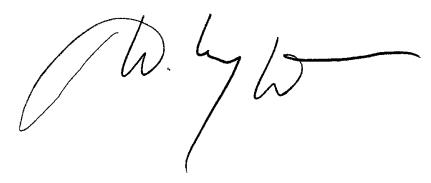

> Die anerkannte klassische Indikation zur Hormonersatztherapie ist das klimakterische Syndrom.

Wie aber kann man vorgehen, wenn klimakterische Symptome bei Frauen mit hormonabhängigen Tumorerkrankungen vorliegen? Alternativen in der Behandlung für diese Patientengruppe zeigen von Wahlde u. Kiesel auf.

Nach den zahllosen Publikationen zu HRT, Pille und Krebs haben die Arbeiten von Frau Brinton vor wenigen Jahren gezeigt, dass auch die Gonadotropinstimulation negative Einflüsse auf die Krebsentstehung hat. Ob dies so in weiteren Studien bestätigt werden konnte, beleuchtet die Übersichtsarbeit von von Horn et al. aus Lübeck.

Wir haben in dieser Ausgabe die übliche Betrachtungsweise, die sich meist auf das Krebsrisiko von Hormonbehandlungen beschränkt, bewusst verlassen und die Zusammenhänge zwischen Hormonen und Krebs aus verschiedenen Blickwinkeln beleuchtet. So können wir Ihnen mit einer einzigen Ausgabe eine ganz aktuelle Grundlage für die Beratung Ihrer Patientinnen an die Hand geben.
W. Küpker
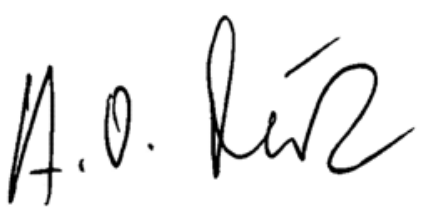

A.O. Mueck

\section{Korrespondenzadresse}

\section{Prof. Dr. T. Strowitzki}

Gynäkologische Endokrinologie und Fertilitätsstörungen, Universitäts-Frauenklinik Heidelberg

thomas.strowitzki@med.uni-heidelberg.de

Interessenkonflikt. T. Strowitzki, W. Küpker und A.O. Mueck geben an, dass kein Interessenkonflikt besteht. 